\title{
Visual analysis of global comparative mapping of the practice domains of the nurse practitioner / advanced practice nursing role in respondent countries
}

Pedro Sastre-Fullana, PhD, MSN, RN (Registered Nurse), Deborah C. Gray, DNP, RN, ANP-BC, FNP-C, FAANP (Associate Graduate Programs Director, Clinical Associate Professor), Andrew Cashin, PhD, MN, BHSC, Dip App Sci, GCert, PTT, GCert, Hpol, RN, NP, MHN, FACNP, FACMHN, FACN (Professor)2, Denise Bryant Lukosius, PhD, RN, CON(C) (Associate Professor), Lorna Schumann, PhD, RN, FNP-C, ACNP-C, ACNS-BC, ENP C, FAAN, FAANP (Commissioner), Franziska Geese, MScN, RN, POC SSPO (Research Associate), Bernadette Rae, MSc, RN (Associate Professor), Elsie Duff, PhD, MEd, BScN, RN, NP (Assistant Professor), \& Beverley Bird, PhD, MPH, MSN, BAppSci, RN, ANP, FACN (Adjunct Lecturer).

\section{Abstract Organize information in sections as in author guidelines}

Background: Internationally, there is increasing demand for nurse practitioner (NP) and advanced practice nursing (APN) roles; however, high variability exists in how NP/APN roles are defined and understood.

Purpose: The aim of this research was to improve our understanding of how the NP/APN is defined globally by: 1) examining role definitions, competencies, and standards of practice for advanced practice nurses internationally; 2 ) describing from a global perspective the core concepts and common features of NP/APN associated with practice domains; and 3) exploring the utility of text mining and visual analytics in identifying the clustered core concepts common to NP/APN roles organized around the five advanced practice domains of the Strong Advanced Practice Model.

Methods: This article describes the findings of a secondary analysis of an international NP/APN competency mapping project, using innovative text mining and visual analysis techniques to reexamine and summarize the NP/APN role in 19 countries from Africa, Australia, Asia, Europe, and North America.

Results: Although weak aggrupation/associations suggest that further work is needed to define the domains of advanced practice with associated model development, visual analysis points to the identification of common concepts and linkages between concepts for each practice domain of advanced practice outlined in the Strong Model.

Implications for practice: The secondary text mining and visual analysis presented in this article allows for comparison of core elements between advanced practice role descriptions, standards, and competencies globally to ultimately provide a global perspective on the common features of NP/APN roles and areas where further delineation is required.

Keywords: Advanced nursing practice domains; advanced practice nurse; advanced practice nursing competency mapping; advanced practice nursing definition; advanced practice nursing role; advanced practice nursing; clinical nurse specialist; global nursing; nurse practitioner; semantic networks; strong model; text mining; visual analytics. 


\section{Introduction}

Internationally, there is increasing demand for nurse practitioner (NP) and advanced practice nursing (APN) roles; however, high variability exists in how NP/APN roles are defined and understood (Heale \& Rieck Buckley, 2015; Hutchison et al., 2014). Many factors have contributed to the inconsistent understanding of these roles globally, including varied country-level health care contexts that affect on the education and regulation of nurses and the stage of APN role development. For that reason, the International Council of Nurses (ICN) (Goodyear, 2008) emphasized a broad and inclusive definition of the NP/advanced practice nurse as a "registered nurse who has acquired the expert knowledge base, complex decision-making skills and clinical competencies for expanded practice, the characteristics of which are shaped by the context and/or country in which $\mathrm{s} /$ he is credentialed to practice." As the demand for and introduction of APN roles increases globally, so too does the need to achieve greater role clarity. Establishing international consensus on APN role definitions, competencies, and standards of practice is necessary to support the optimal development and utilization of these roles to meet global priorities for improving health (Bryant-Lukosius \& MartinMisener, 2016). This article describes the findings of a secondary analysis of an international map ping project using text mining and visual analysis to determine the common features of APN roles in 19 countries. For the purposes of this project, APN was defined as the role descriptions, standards of practice, and competencies that characterized this area of practice in each participating country. Project findings can be used to make recommendations about the consistent features of APN and areas where further delineation is required

\section{Background}

The core focus of the International Council of Nurses Nurse Practitioner/Advanced Practice Nurse Network (ICN NP/APNN) Research Subgroup (RSG) is to gather, analyze, and report data on the development of APN worldwide to support practice, research, and policy (ICN NP/APNN, 2019). Research Subgroup members participating in the 2013 ICN International Congress in Melbourne, Australia recognized the need to assess the current state of APN globally by examining formal country-level documents related to role definitions, competencies, and scope of practice. Through this examination, diverse and common areas of agreement on how APN is defined internationally could be determined as the basis for establishing priorities to improve role clarity. At the time, the North American and international focus of APN was on the two dominant APN frameworks represented by the ICN publication on APN scope of practice (Goodyear, 2008) and a conceptual model, the Mick and Ackerman, 2000 Strong Model of Advanced Practice. The NP/APN Comparison Mapping Project and ICN NP/APNN Research Subgroup (2019) mapping document presented those findings, providing an international perspective on the NP/APN through a global cross-mapping of roles and competencies of advanced practice nurses.

This research focuses on a secondary analysis of the previous ICN NP/APNN Research Subgroup cross-mapping results to further evaluate the information in the larger 186-page published summary document. The Strong Advanced Practice Model (Mick \& Ackerman, 2000) was selected as the organizational framework for this project because of its focus on the five domains of practice that are commonly used by APNs in both acute and primary care settings (Arslanian-Engoren, 2018) and its ability to adjust to the variations in recognized roles in international com munities who have ANP roles (Carryer et al., 2017; Gardner et al., 2016). The five advanced practice domains of the Strong Model are direct comprehensive care, education, support of systems, research, and publication and 
professional leadership, with each domain containing a wide range of focus areas and activities (Mick \& Ackerman, 2000).

The aim of this research project was to improve our understanding of how the APN is defined around the world by achieving these specific objectives to: 1) examine role definitions, competencies, and standards of practice for advanced practice nurses internationally; 2) describe from a global perspective the core concepts and common features of APN associated with the five practice domains; and 3) explore the utility of text mining and visual analytics in identifying the clustered core concepts common to APN roles organized around the five advanced practice domains of the Strong Model for conceptual coherency. This article describes the mapping project, with a specific focus on the results of secondary visual analysis of the data to provide a global perspective on the common features of APN roles

\section{Introduction to text mining and visual analytics}

Text mining and visual analytics are innovative techniques that were used in this project to summarize and evaluate large amounts of text data extracted from country-level documents on APN. The visual analysis presented in this article is based on specific methods of text mining and natural language processing (Tan, 1999) to identify elements that are closely related. These methods include first identifying words as single elements (called tokenization) or sentences, filtering stop words, punctuations, and numbers (Vijayarani et al., 2015). Subsequently, for a proper visual analysis of text data, modularity-based clustering algorithms (Madhulatha, 2012) based on word frequency counting, and co-occurrence of terms in a sentence or paragraph, were then applied to measure the strength of division of a network into clusters (or groupings). A connection degree score was also calculated, based on the total number of connections that a node (usually a word or small words sequence) has to other nodes or words in the network. The connection degree scores are a direct indicator of its importance in the network and as a core concept in the text data (Martin et al., 2014).

Equally important are other analyses or measures of centrality. The Centrality score (Jamour et al., 2017) identifies key terms (nodes that are the midpoints between two other nodes) that serve as a conceptual bridge between many other connected elements within a network or one of its clusters or groupings. This score highlights core concepts for the whole text. Another analysis used in this process is calculation of the Eigenvector Centrality score (Bonacich, 2007), which identifies underlying elements that have the potential to influence other elements of the network/cluster.

The visual analysis in this article is also represented by Semantic Network Graphs that illustrate the sematic relationships between concepts in a network from the text mining and analysis. In the graph, every element (word or term) is represented as a node. Linked nodes (this specific linkage is represented as an edge) conform to a single cluster that has some conceptual significance. The overall graph consists of vertices, which represent concepts, and edges, which represent semantic relationships between concepts. The final shape is a rooted tree that represents a "conceptual graph" or diagram that summarizes the information in the text analyzed and aids in understanding in a glimpse the core elements of the original mapping results. See the explanatory image to better understand the components of visual analysis (Figure 1). 


\section{Methods}

Data collection The RSG co-chairs, in conjunction with the subgroup members developed a proposal for submission to the ICN Network Core Steering Group (CSG) for review and sub sequent approval. The main focus of data collection in the original study was on documents outlining APN role descriptions, policies, standards of practice, or competencies that were officially recognized or produced by national nursing associations, nursing regulatory boards, or ministries of health in each country. Data were collected from grey literature found on nursing and government websites and from ICN NP/APN RSG members and key stakeholders in countries willing to participate. Research Subgroup members collected the information relevant to their own country and enlisted to the help of reliable colleagues to collect data in countries with no RSG member. The data were collected between January 2014 and December 2017. International Council of Nurses regulations during this period prohibited implementation of a global survey to collect the data.

Sample The sample included information contained in the 186- page published mapping document (ICN NP/APNN Research Subgroup, 2019) on advanced practice nurses in generically titled roles, clinical nurse specialists, and NPs in 19 countries. The countries included Australia, Botswana, Canada, China, England, Finland, Germany, Japan, Macau, Netherland, New Zealand, Republic of Ireland, Scotland, Singapore, Spain, Switzerland, Norway, United States of America, and Wales.

Analysis This article presents the results of an ICN-approved secondary analysis of an original APN Mapping Project in which NP/APN role descriptions, policies, competencies, standards, and other pertinent information was collected from 19 countries drawn from Africa, Australia, Asia, Europe, and North America. The role description and competency areas and indicators for each of the roles were then compiled for each country into an international comparative role-specific table mapped with both the Strong Model and the ICN APN model domains of practice. The resulting 186-page mapping document was then submitted to ICN and published on the ICN NP/APN Network webpage (ICN NP/APNN Research Subgroup, 2019). The pattern relations between terms in this resulting document provided the basis for the visual analysis presented.

The secondary analys is, which is the basis for this research, was then done to evaluate the data in the larger mapping document to look for common conceptual strands across domains of practice for the NP/APN roles. All methods used in this secondary analysis are based on the processing capabilities of Gephi software application. Data management involved in-putting the terms from all countries to examine visual patterns using visual analytics Gephi software (Bastian et al., 2009). Using text mining and visual analytics, the underlying clustered core concepts common to global APN roles were identified and grouped by key elements of the advanced practice domains of the Strong Model for conceptual coherency.

Results The average clustering coefficient (Table 1 ) ranged from 0.279 to 0.322 , which represents a relatively weakgrouping of the concepts represented in the Strong Model present in the data. However, the composite scores obtained from this analysis must be understood from the semantic field relations rather than from a model/theory concordance process. Thus, the clustering coefficients reflect more the level of grouping of concepts and their relationships in the original 186page mapping document rather than with the model. The analysis suggests that each domain is a consistent set of interconnected elements that tends to group coherently into related subgroups of 
items, with no extreme variation (indication of a coherent back ground) between its different domains (Newman, 2016).

Visual analyses In the visual analyses, graphic representation of semantic networks within the larger textual mapping document allows them to be visualized and inferences drawn regarding relationships between concepts or terms in each domain. In this visual representation of networks, the se mantic concepts or nodes are represented by circles, and the links or the clockwise curved lines are the conceptual connection between nodes [concepts; terms]. The more connections a node has to other nodes, the larger its circle appears, and the higher strength of a connection is also represented with thicker lines. Clusters are clearly represented by specific colors in the graph, one for every cluster that has been identified in the analysis process. Using this analysis and graphic process, the following figures and discussion represent and summarize the results in relation to each of the five advanced practice domains.

Direct comprehensive care advanced practice nursing domain. Direct comprehensive care visual analysis is rep resented in Figure 2 and included 104 words that clustered around 8 main groups, with the key elements of each one being care, knowledge, family, patient, and client (Figure 2). The analysis also identified connective-bridging terms as represented by the clustering coefficient (high scoring concepts from the betweenness centrality measure) that bring cohesion to the whole semantic network. In the direct care domain, these bridging concepts are patient outcome, response, and decision making. Surrounding those elements, there were several other terms that were directly connected to key terms (measured through eigenvector centrality methods) including therapeutic intervention and skill.

Education advanced practice nursing d omain. Education visual analysis is represented in Figure 3 and comprised a total of 113 words and 9 clusters in which the node degree approach highlights in order of importance health consumer; participate; practice; community: health care; preceptor; and knowledge as core elements of this domain (Figure 3). The connective-bridging terms or high scoring concepts in this domain are diagnosis and quality, and other concepts directly connected to key these terms are teaching and support.

Support of systems advanced practice nursing domain. Support of systems visual analysis is represented in Figure 4 and encompassed 11 clusters for a total of 117 words with the primary items from node degree scoring being leadership, practice, service, health outcome, quality, patient, and professional organization (Figure 4). Evaluation, care provider, and community are the high scoring bridging concepts, and effectiveness and evaluation are the primary concepts related to these terms.

Research advanced practice nursing domain. Research visual analysis is represented in Figure 5 and identified 8 clusters with evidence; practice and research; outcome; new knowledge being the focus items based on node degree analysis from a total amount of 110 elements of this domain (Figure 5). The connective-bridging terms or high scoring concepts in this domain are organization, individual, and plan. Other concepts highly connected to these terms are value and service.

Publication and professional leadership advanced practice nursing domain. Publication and professional leadership visual analysis is represented in Figure 6 and contained 114 words. The analys is shows 10 clusters with the next critical nodes: leadership; practice; care; evaluation; professional context and critique health care policy; partnership and system; responsibility; and 
educator (Figure 6). Development, educator, and advocate are the bridging highest scoring terms in this domain, with conflict resolution skill and guide connected to these terms.

Discussion There is heightened activity in many countries to delineate or further refine definitions of competencies and standards to clarify and strengthen the implementation of APN roles (BryantLukosius \& Wong, 2018). However, determining key commonalities specific to APN roles remains complicated (Sastre-Fullana et al., 2014). As part of the earlier phase of this project, the role description and competency areas and indicators for each of the APN roles were compiled for respondent countries into an international comparative role-specific table mapped with both the Strong Model and the ICN model domains of advanced practice.

The 186-page document (ICN NP/APNN Research Subgroup, 2019) serves as a compendium of role descriptions and different levels of competencies that are specific for APNs in different countries. However, the correlation and correspondence of the selected competencies had yet to be evaluated and discussed. The secondary text mining and visual analysis presented in this article allows for comparison of core elements between advanced practice role descriptions, standards, and competencies globally. Although weak aggrupation/associations suggest that further work is needed to define the domains of advanced practice with associated model development, visual analysis points to the identification of common concepts and linkages between concepts for each domain of advanced practice outlined in the Strong Model. This finding is consistent with review of APN competency frameworks conducted by Sastre-Fullana et al. (2014) that found agreement on 17 competencies across six types of roles in 26 countries. In the direct comprehensive care domain assessment, diagnosis, evidence-based practice, direct care, and critical thinking are clearly identified in the Semantic Network that belongs to this domain. Cluster analysis points toward evidence-based and client-centered care as core elements and that are congruent with concepts developed by the Strong Model. Other important items, such as "therapeutic intervention," "knowledge," and "skill" act as linking points between other key elements in this domain, where direct care is the main core concept. This idea points toward instrumental abilities and acquaintance that reflect the essence of clinical practice (Nieminen et al., 2011) and specific procedures. Also, communication was identified in the network as a transversal part of all processes with other professionals, patients, and families.

The education domain visual analysis clearly indicates that a key part of the whole process is the health consumer and identifies the teaching, coaching, learning, and decision-making processes that are key to the patient education concept through participation and implication in his health-illness continuum (Scarpa \& Connelly, 2011). Preceptorship and mentoring of other professionals appear as interconnected essential concepts of the Education semantic network and is consistent with this component of APN globally (Arslanian-Engoren, 2018). Within the identified semantic network, the terms that bring coherency to the entire network are teaching that is implicitly related to all other elements, and support due to the complexity and additional elements that a truly effective education process requires (Lindblad et al., 2010). The complexity of support of systems domain is transversal to the other domains and closely related to their key elements due to consultation processes needed in managing health resources to meet priorities of care, along with strategic planning, innovation, and change management, although maintaining quality improvement (Brooten et al., 2012). At the centre of the entire network, professional practice is found as the axis around which other elements connect, in clearly defined clusters that merge leadership, service, health 
outcomes, patient, implementation, and professional organization core concepts (Hutchinson et al., 2014). The research domain offers a vision of the principles related to the close relation between research and evidence-based practice that is a pillar of the advanced nursing practice and its different roles (DeNisco \& Barker, 2012). Around those core concepts, a myriad of elements emerge that are coherently distributed and connected to provide understanding for the generation of new knowledge and improving patient and professional outcomes. The duality of the core concepts related to the publication and professional leadership domain are well illustrated in the visual analysis results where practice is an essential part of the conceptualization and is clearly intertwined with leadership in order of importance. Clinical and professional leadership is a well-known and extensively discussed crucial component of advanced practice (Elliott et al., 2016). Publicationrelated concepts (Mateo, 2013) seem more dispersed and less established in the competency maps.

Conclusions Visual analysis tools add new opportunities for text mining and semantic networks analysis of documents. This analytic approach was used to examine conceptual coherency of country-related APN documents, in relation to an established framework outlining domains of advanced practice. Although the clustering coefficient or aggrupation to the Strong Model domains of practice was relatively weak, this may be the result of the variety of document types included. If the mapping was restricted to competency documents, it is plausible that the aggrupation or aggregation may have been stronger, and this warrants further research. Of note, the data did aggregate in a manner that established that leadership and active participation in policy development are a focus and part of practice of advanced practice nurses.

Further research is indicated to explore development of models that would encapsulate the $68 \%$ of data mapped in the documents that did not form part of the semantic networks identified in the visual analysis. It is plausible that the model used in the analysis has a close fit with competency documents included, the domain for which it was designed, but less of a fit with standards for practice and other types of documents related to APN practice. An analysis of the $68 \%$ of data that did not aggrupe with the Strong Model concepts will be as valuable as the extra dimension revealed from the 32\% that did aggrupe with the Strong Model. Arguably, knowledge of the diversity of APN practice will be gleaned as much, from what does not fit with the existing model used deductively in the current project.

The purpose of this analysis was to describe an international mapping project of APN definitions, competencies, and scope of practice with a specific focus on the results of secondary visual analysis summarizing the extensive data, to provide a global perspective on the common features of APN roles and areas where further delineation is required. The findings from this project further highlight the wide variability in the advanced practice nurse globally that has not only added to confusion regarding the APN but also potentially limited development and implementation of the role and its potential benefits for vulnerable patient populations as well as the nursing profession. To this end, the ICN, in recognizing the need for greater clarity, is drafting a new guidance paper on the APN, updating the general 2002 APN definition with specific recommendations and guidelines to address APN terminology, education, scope of practice, and role implementation (International Council of Nurses, 2020; Rodgers, 2019; Schober \& Stewart, 2019). This guidance may affect this significant variability and may pave the way for future APN practice model development. 


\section{References}

Arslanian-Engoren, C. (2018). Conceptualizations of advanced practice nursing. In: M. M.F. Tracy \& T T.O'Grady(Eds.). Advanced practice nursing: An integrative approach (6th ed., pp. 25-60). Elsevier.

Bastian, M., Heymann, S., \& Jacomy, M. (2009). Gephi: An open source software for exploring and manipulating networks. Third In ternational AAAI Conference on Weblogs and Social Media, pp. 361-362. https://doi.org/10.1136/qshc.2004.010033.

Bonacich, P. (2007). Some unique properties of eigenvector centrality. Social Networks, 29, 555-564.

Brooten, D., Youngblut, J. M., Deosires, W., Singhala, K., \& Guido-Sanz, F. (2012). Global considerations in measuring effectiveness of advanced practice nurses. International Journal of Nursing Studies, 49, 906-912.

Bryant-Lukosius, D. \& Martin-Misener, R. (2016). Advanced practice nursing: An essential component of country level human resources for health. International Council of Nurses Policy Brief. ICN.

https://www.who.int/workforcealliance/knowledge/resources/ICN_PolicyBrief6AdvancedPracticeNu rsing.pdf.

Bryant-Lukosius, D., \& Wong, F. K. Y. (2018). Global development of advanced practice nursing. In: M. M.F. Tracy and T T.O'Grady(Eds.). Advanced practice nursing: An integrative approach (6th ed., pp 129-141). Elsevier.

Carryer, J., Wilkinson, J., Towers, A., \& Gardner, G. (2017). Delineating advanced practice nursing in New Zealand: A national study. International Nursing Review, 65, 24-32.

DeNisco, S. M. \&. Barker, A. M. (2013). Advanced practice nursing: Evolving roles for the transformation of the profession (2nd ed.). Jones and Bartlett Publishers.

Elliott, N., Begley, C., Sheaf, G., \& Higgins, A. (2016). Barriers and enablers to advanced practitioners' ability to enact their leadership role: A scoping review. International Journal of Nursing Studies, 60, 24-45.

Gardner, G., Duffield, C., Doubrovsky, A., \& Adams, M. (2016). Identifying advanced practice: A national survey of a nursing workforce. In ternational Journal of Nursing Studies, 55, 60-70.

Goodyear, R. T. (2008). The scope of practice, standards, and competencies of the advanced practice nurse: ICN regulation series. International Council of Nurses.

Heale, R. \& Rieck Buckley, C. (2015). An international perspective of advanced practice nursing regulation. International Nursing Review, 62, 421-429.

Hutchinson, M., East, L., Stasa, H., \& Jackson, D. (2014). Deriving consensus on the characteristics of advanced practice nursing: Metasummary of more than 2 decades of research. Nursing Research, 63, 116-128.

International Council of Nurses. (2020). Guidelines on advanced practice nursing 2020. https://www.icn.ch/system/files/documents/2020-04/ICN_APN\%20Report_EN_WEB.pdf. International Council of Nurses NP/APN Network. (2019). Research: Focus of subgroup. http://icnapnetwork.org/. 
International Council of Nurses NP/APN Network Research Subgroup. (2019). Comparison of the strong model of advanced practice nursing and the International Council of Nurses (2008) advanced practice nursing competencies with advanced practice nursing competencies from respondent countries 2014-2017. http://international.aanp.org/Content/docs.

Jamour, F., Skiadopoulos, S., \& Kalnis, P. (2017). Parallel algorithm for incremental betweenness centrality on large graphs. IEEE Transactions on Parallel and Distributed Systems, (pp. 1). https://doi. org/10.1109/TPDS.2017.2763951.

Lindblad, E., Hallman, E. B., Gillsjö, C., Lindblad, U., \& Fagerström, L. (2010). Experiences of the new role of advanced practice nurses in Swedish primary health care-A qualitative study. International Journal of Nursing Practice, 16, 69-74.

Madhulatha, T. S. (2012). An overview of clustering methods. IOSR Journal of Engineering, 2, 719725.

Martin, T., Zhang, X., \& Newman, M. E. J. (2014). Localization and centrality in networks. Physical Review E - Statistical, Nonlinear, and Soft Matter Physics, 90, 52808.

Mateo, M. A. (2013). Research for advanced practice nurses: From evidence to practice. Springer Publishing Company.

Mick, D. J., \& Ackerman, M. H. (2000). Advanced practice nursing role delineation in acute and critical care: Application of the strong model of advanced practice. Heart \& Lung: The Journal of Acute and Critical Care, 29, 210-221.

Newman, M. E. J. (2016). Equivalence between modularity optimization and maximum likelihood methods for community detection. Physical Review E, 94, 052315.

https://doi.org/10.1103/PhysRevE.94.052315.

Nieminen, A. L., Mannevaara, B., \& Fagerstrom, L. (2011). Advanced " practice nurses' scope of practice: A qualitative study of advanced clinical competencies. Scandinavian Journal of Caring Sciences, 25, 661-670.

Rodgers, M. (2019). Advanced practice globally: Where are we now and what does the future hold? [power point presentation]. ICN Con gress 2019, June, Singapore.

Sastre-Fullana, P., De Pedro-Gomez, J. E., Bennasar-Veny, M., Serrano-Gallardo, P., \& MoralesAsencio, J. M. (2014). Competency frameworks for advanced practice nursing: A literature review. International Nursing Review, 61, 534-542.

Scarpa, R., \& Connelly, P. E. (2011). Innovations in performance as sessment: Acriterion based performance assessment for advanced practice nurses using a synergistic theoretical nursing framework. Nursing Administration Quarterly, 35, 164-173.

Schober, M., \& Stewart, D. (2019). Developing a consistent approach to advanced practice nursing worldwide. International Nursing Review. 66, 151-153.

Tan, A. H. (1999). Text mining: The state of the art and the challenges. Proceedings of the PAKDD 1999 Workshop on Knowledge Discovery from Advanced Databases, 8, pp. 65-70. 
Vijayarani, S., Ilamathi, M. J., \& Nithya, M. (2015). Preprocessing tech niques for text mining-an overview. International Journal of Computer Science \& Communication Networks, 5, 7-16

Authors' contributions: In addition to overall review and editing each listed author below also specifically did the following: P. Sastre-Fullana developed idea and per formed all analysis, co-wrote sections on text-mining/visual analysis, results, discussion, and developed all figures and tables. D. C. Gray co-wrote abstract, introduction, background, text-mining/visual analysis section, data collection, sample, analysis, results, discussion, conclusions sections, references, and assisted with table and figure development; served as manuscript coordinator and journal corresponding author. A. Cashin co-wrote introduction, results, discussion, and conclusions sections. D. Bryant-Lukosius cowrote introduction and background sections, and assisted with writing discussion. L. Schumann assisted with writing background, data collection, sample, and analysis section. B. Bird assisted with writing background and data collection sections. F. Geese assisted with methods, idea development, and writing abstract section. E. Duff assisted with performing data analysis. B. Rae assisted with research for literature review for introduction and back ground sections. 
Table 1

\begin{tabular}{lcccccc}
\hline & $\begin{array}{c}\text { Number of } \\
\text { Clusters }\end{array}$ & $\begin{array}{c}\text { Strong } \\
\text { Connected } \\
\text { Components }\end{array}$ & $\begin{array}{c}\text { Average } \\
\text { Degree }\end{array}$ & Modularity & $\begin{array}{c}\text { Average Path } \\
\text { Length }\end{array}$ & $\begin{array}{c}\text { Average } \\
\text { Clustering } \\
\text { Coefficient }\end{array}$ \\
\hline Domain 1 & 8 & 101 & 12,317 & 0,346 & 1,811 & 0,279 \\
Domain 2 & 9 & 106 & 6,587 & 0,572 & 2,147 & 0,32 \\
Domain 3 & 11 & 113 & 8,159 & 0,507 & 2,041 & 0,286 \\
Domain 4 & 8 & 105 & 6,448 & 0,383 & 2,025 & 0,318 \\
Domain 5 & 10 & 112 & 5,661 & 0,569 & 2,197 & 0,322 \\
\hline
\end{tabular}

Table x.1 Main results of graph analysis for the textual corpus of the documents, disaggregated per domains

\section{Main Visual Analysis}

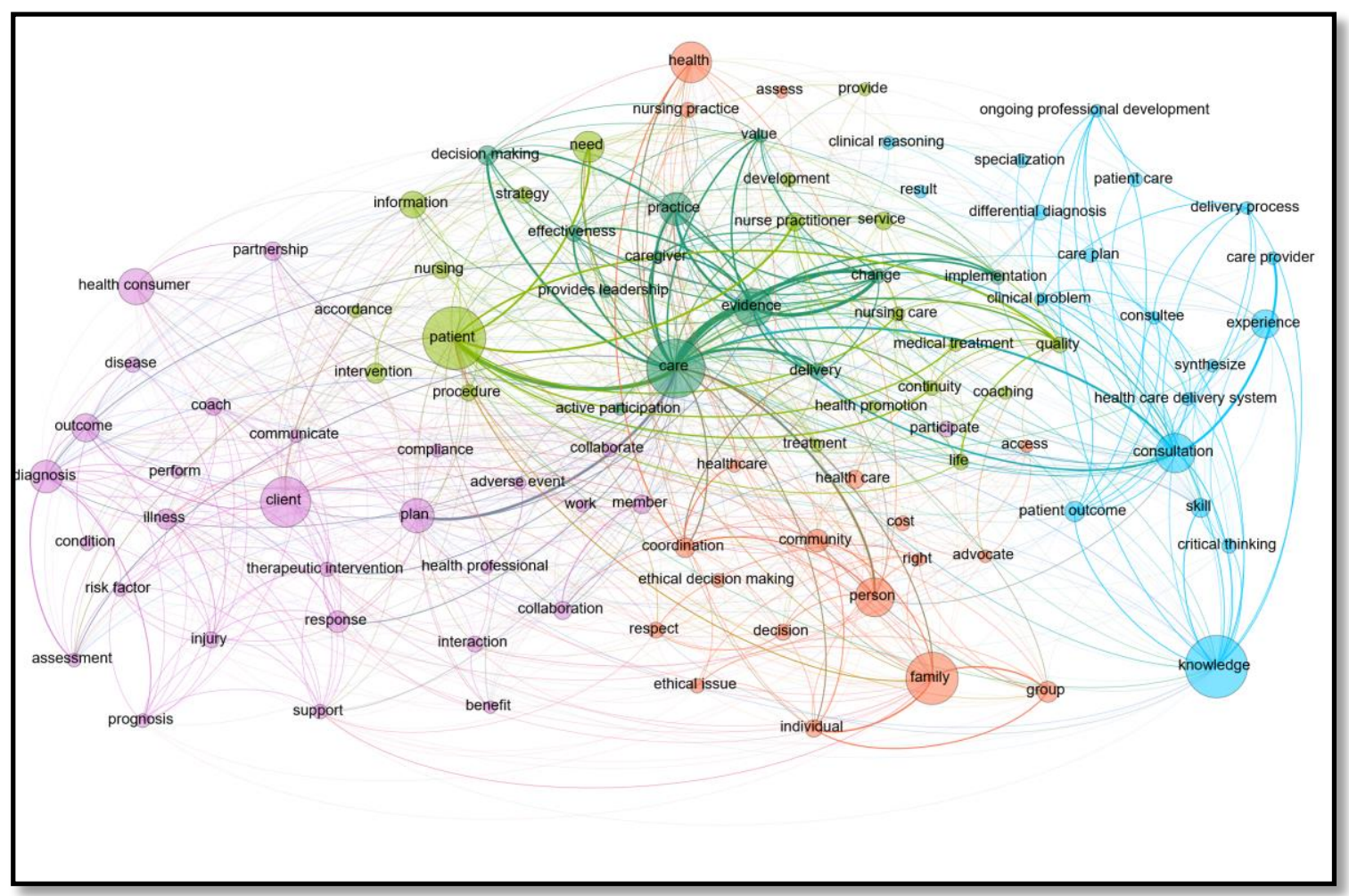

Image 1. Domain 1 Main Visual Analysis 


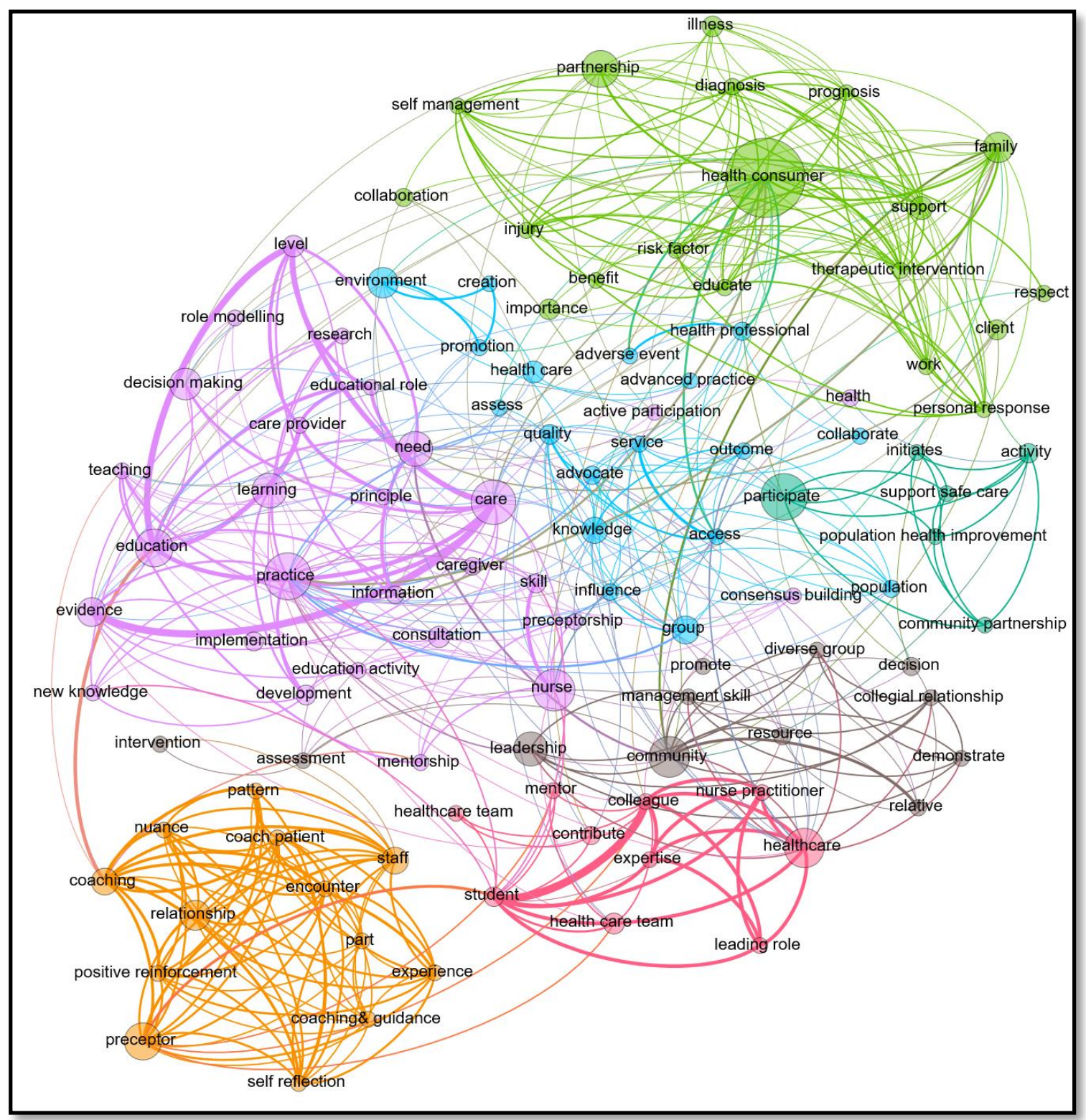

Image 2. Domain 2 Main Visual Analysis 


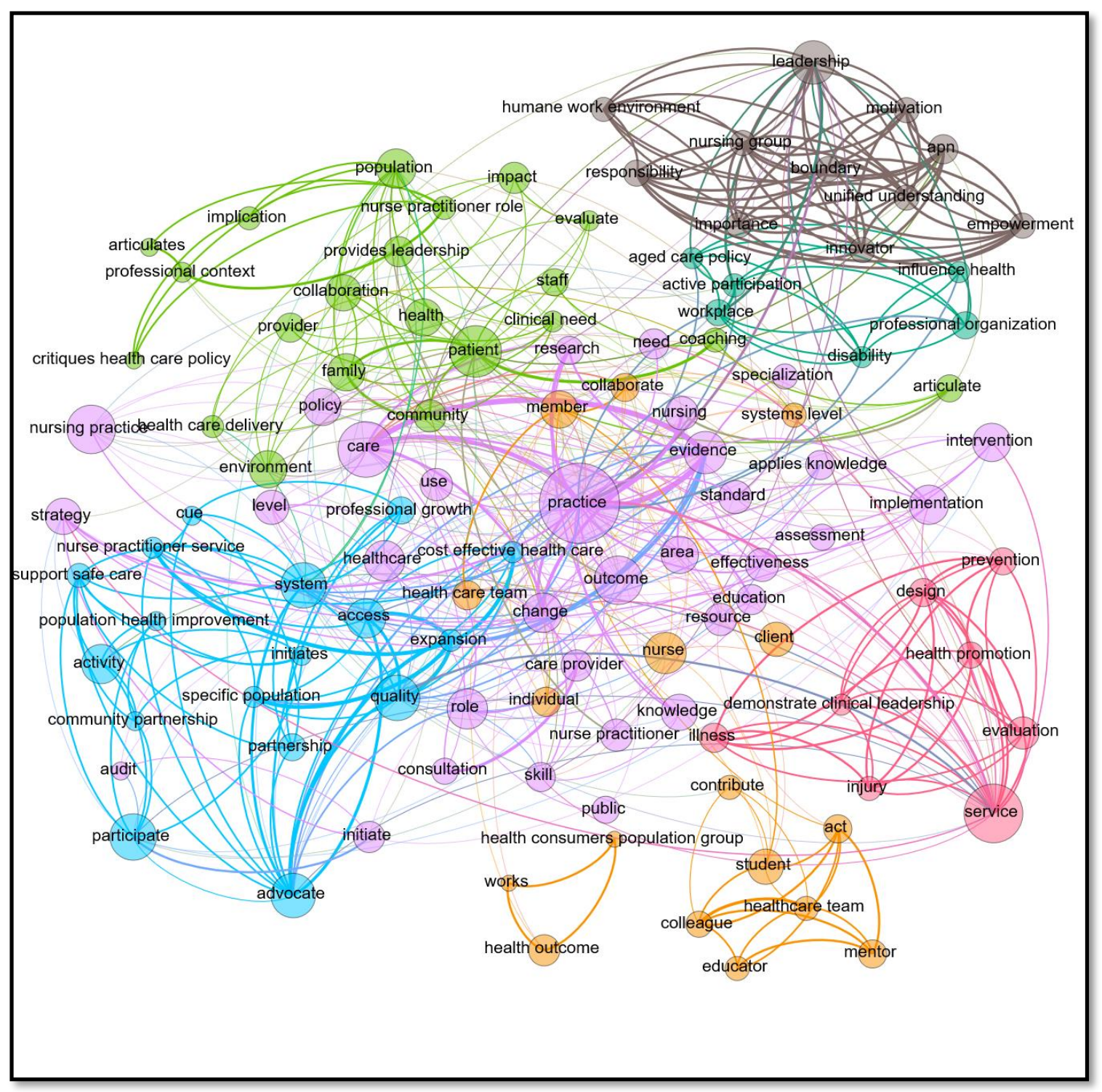

Image 3. Domain 3 Main Visual Analysis 


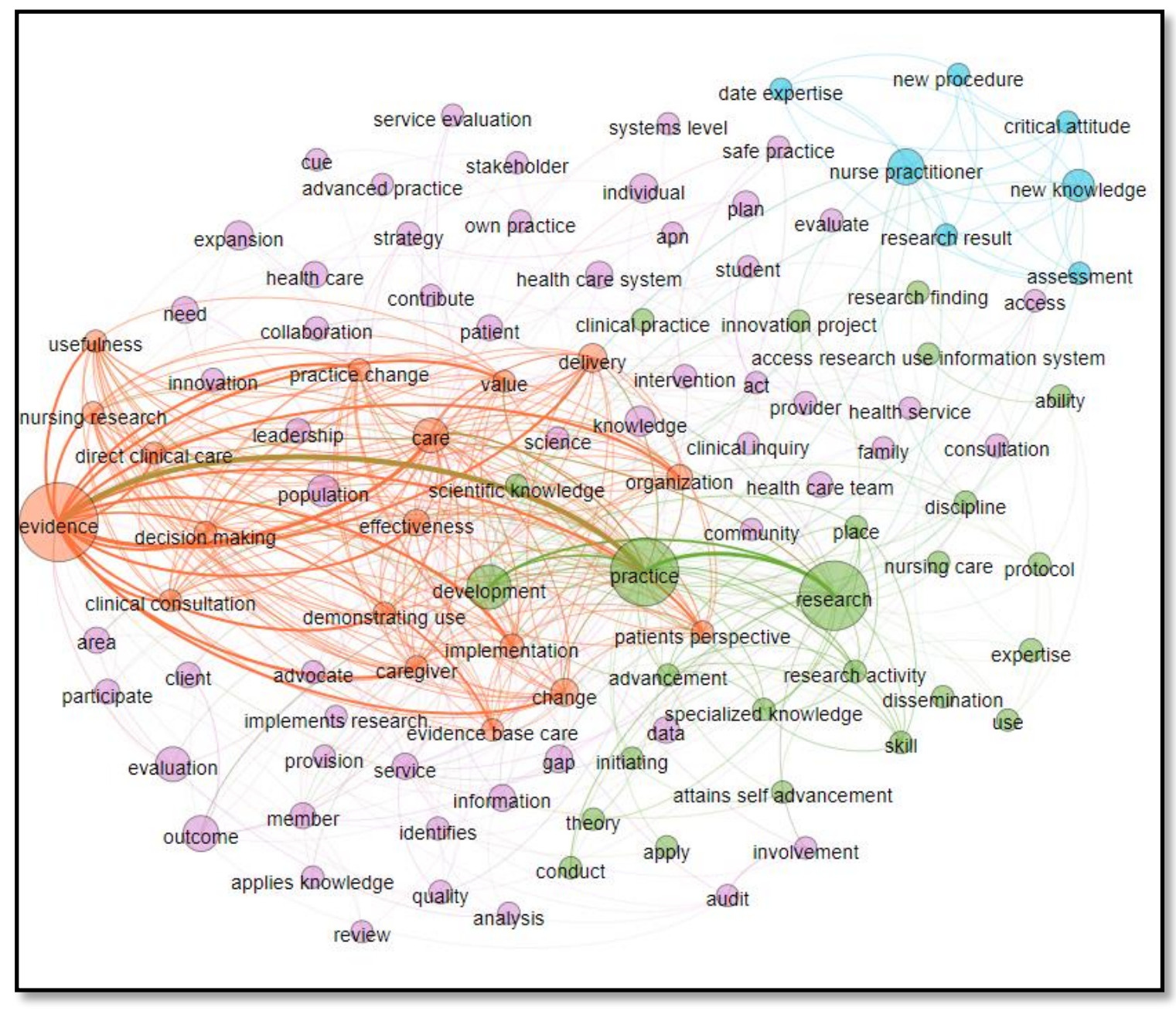

Image 4. Domain 4 Main Visual Analysis 


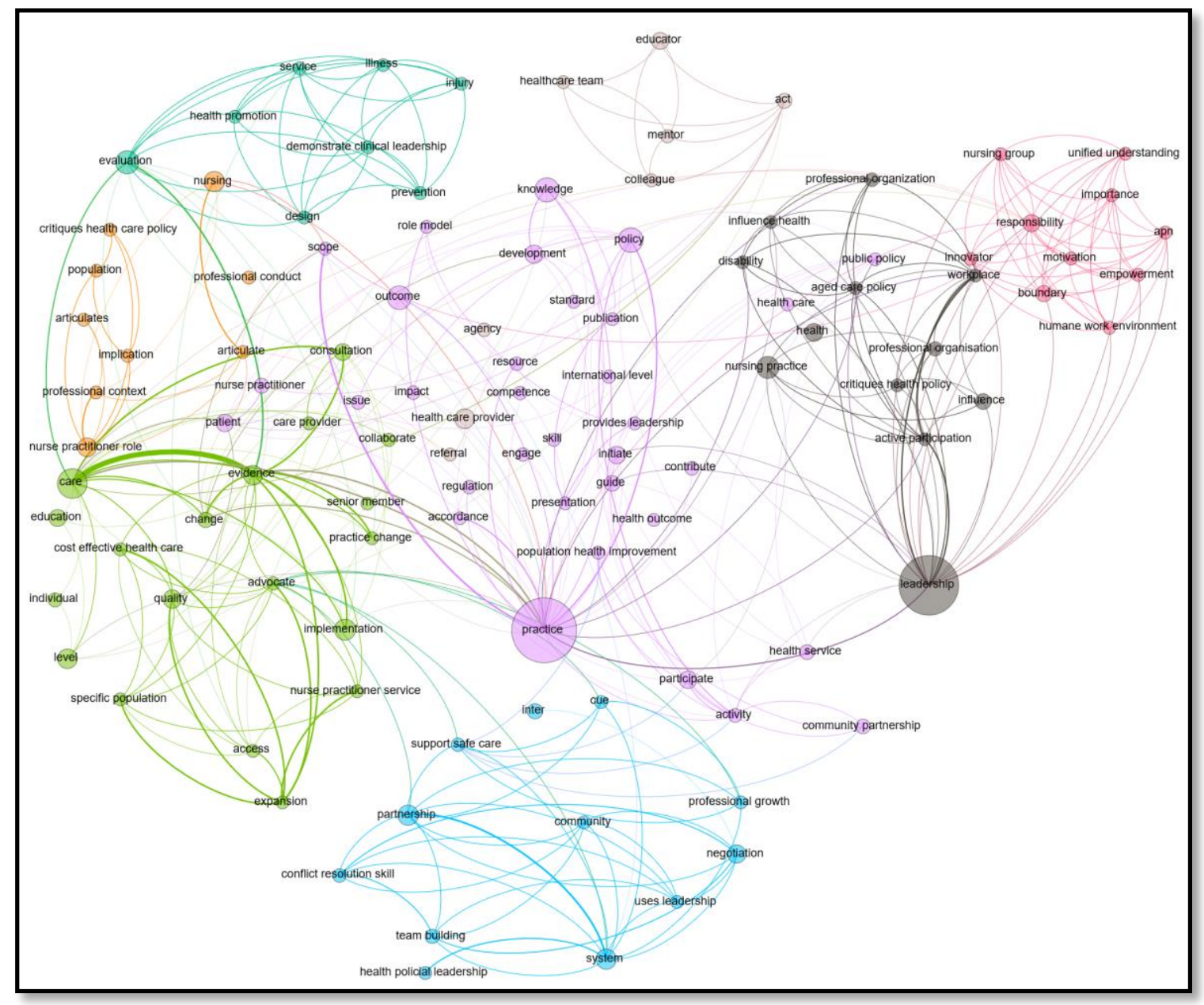

Image 5. Domain 5 Main Visual Analysis 

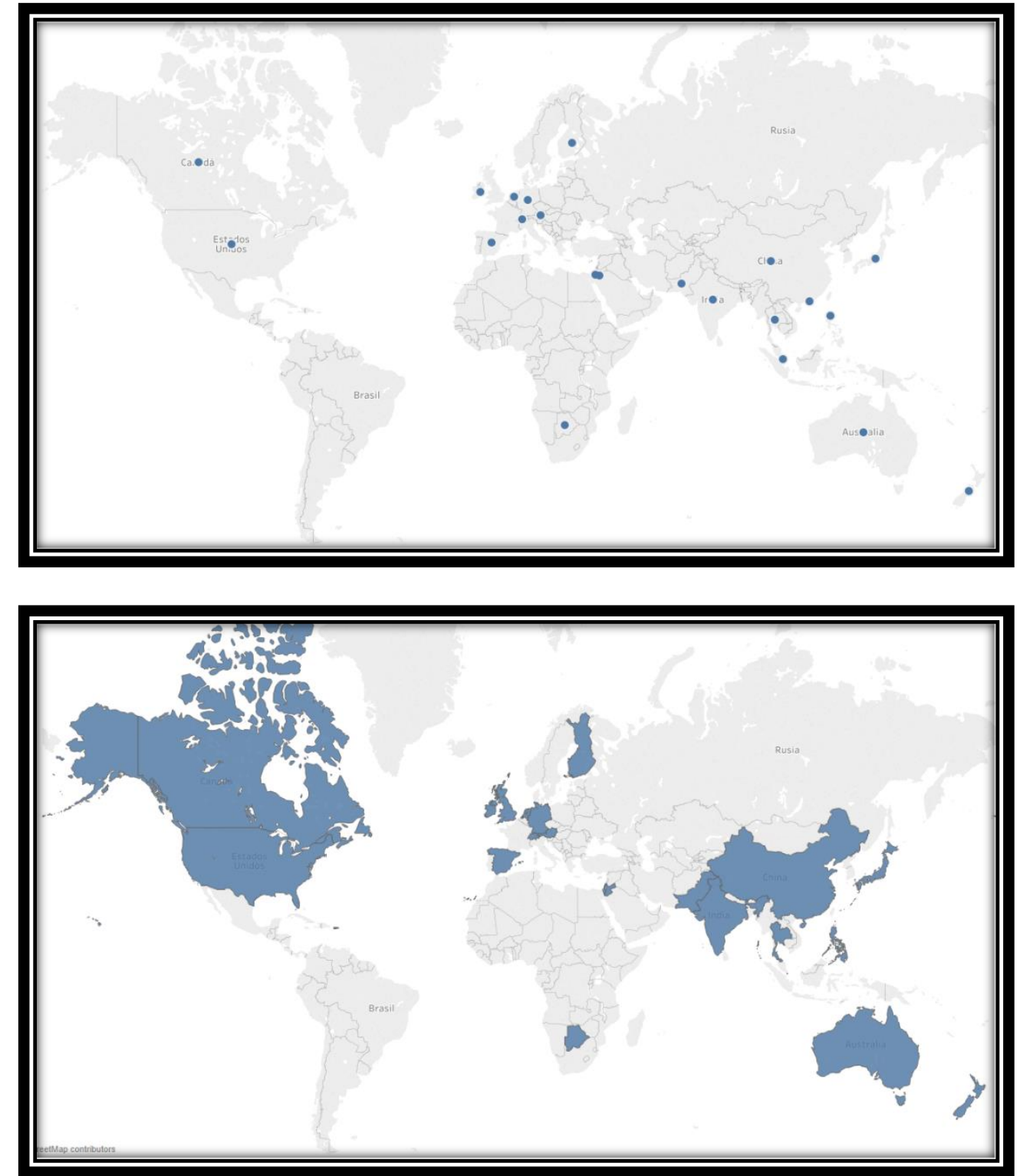

community

health consumer information plan assessment

nursing care evidence care diagnosis group

individual collaborationfamily ${ }^{\text {illness }}$

patient outcome $e_{\text {cordinationon }}^{\text {prace }}$

$\underset{\text { implementation }}{\text { conson }}$ response patient injury decision making ${ }^{\text {support }}$

outcome quality knowledge delivery

therapeutic intervention client change

Image x.1. Core elements of Betweeness centrality for Domain 1 


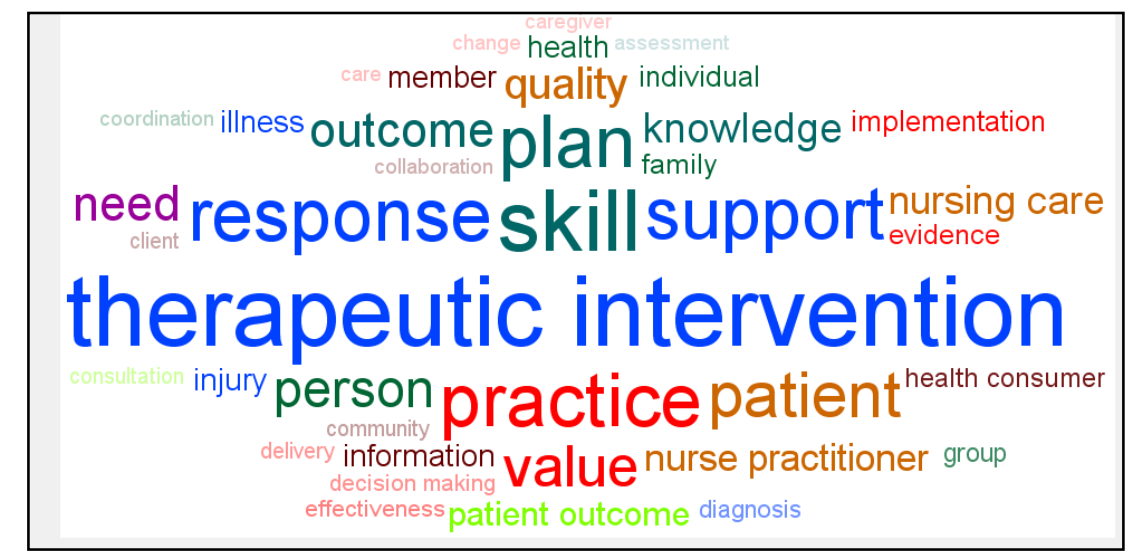

Image x.2. Key elements of Eigenvector centrality for Domain 1

Domain 2: Education, comprises a total of 113 words and 9 clusters in which the Node Degree approach highlights Health Consumer; Participate; Practice; Community: Healthcare; Preceptor; Knowledge as core elements of this domain.

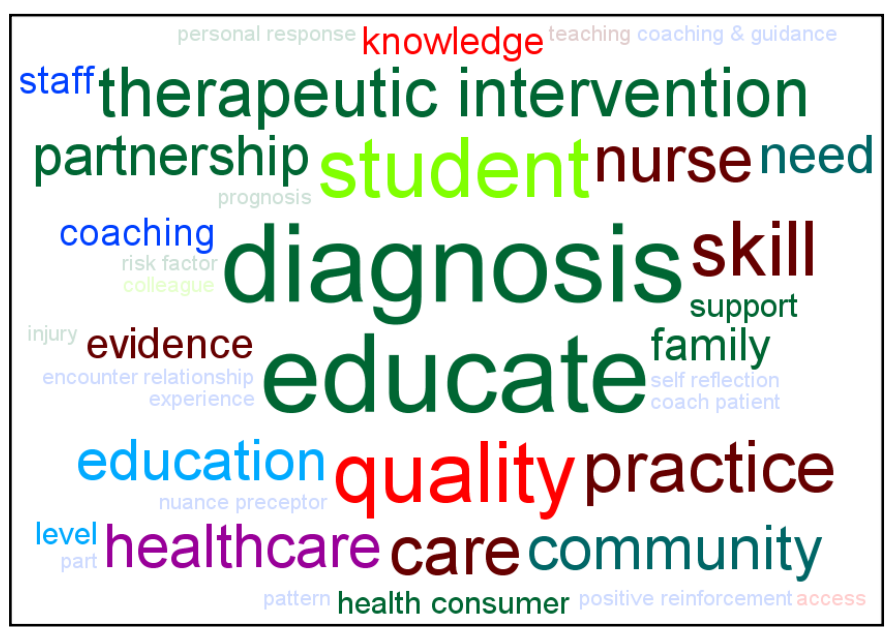

Image x.3. Core elements of Betweeness centrality for Domain 2 


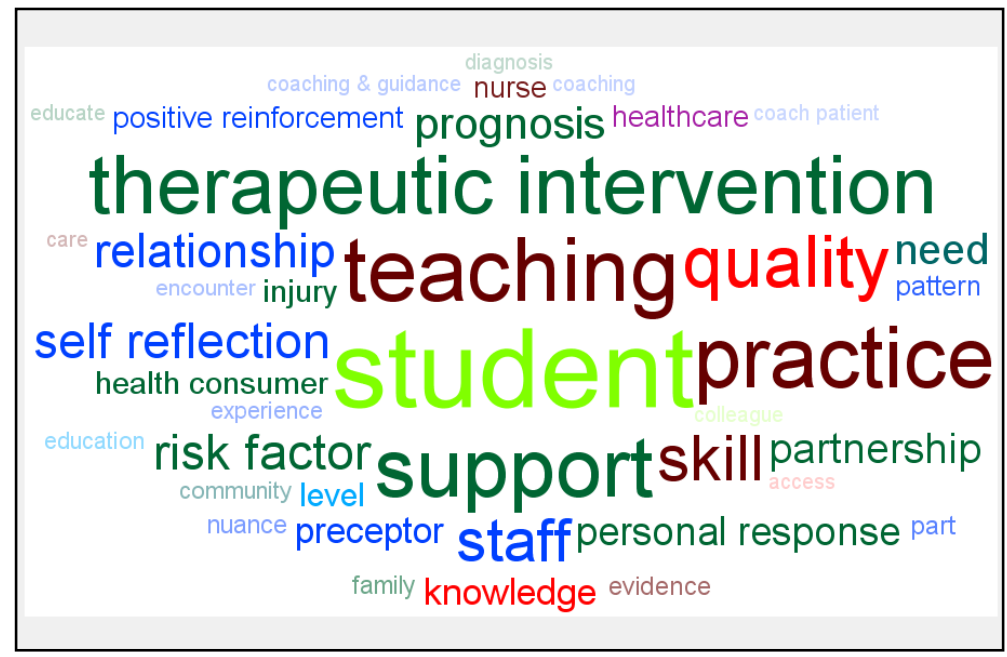

Image x.4. Key elements of Eigenvector centrality for Domain 2

Domain 3: Support of Systems encompasses 11 clusters for a total of 117 words with the main items from Node Degree scoring being Leadership; Practice; Service; Health Outcome; Quality; Patient; Professional Organization.

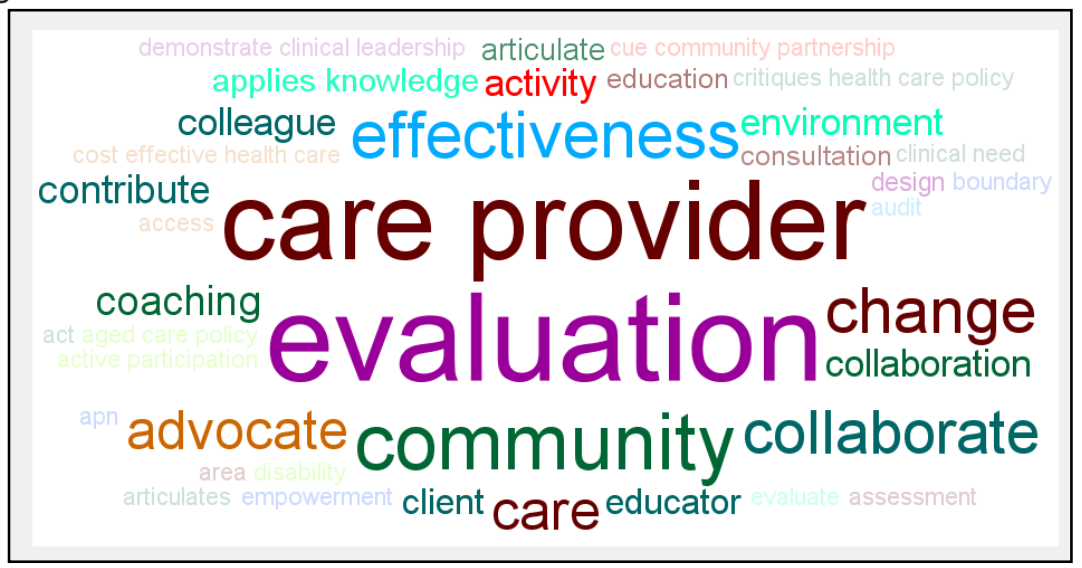

Image x.5. Core elements of Betweeness centrality for Domain 3

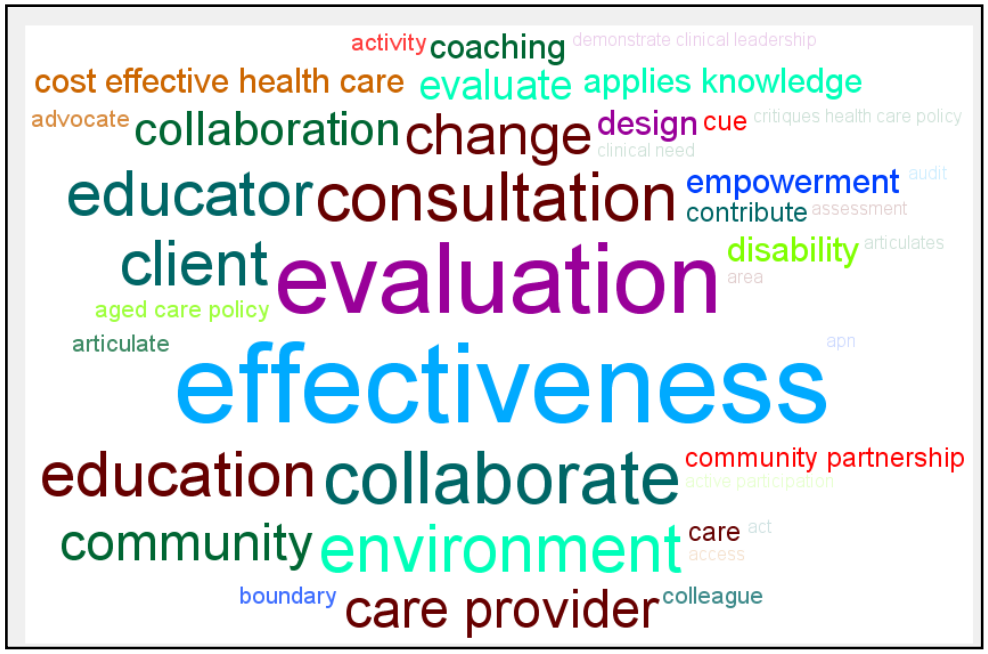

Image x.6. Core elements of Eigenvector centrality for Domain 3 
In Domain 4: Research, 8 clusters were identified, and Evidence; Practice and Research; Outcome; New Knowledge being the focus items based on Node Degree analysis from a total amount of 110 elements of this Domain.

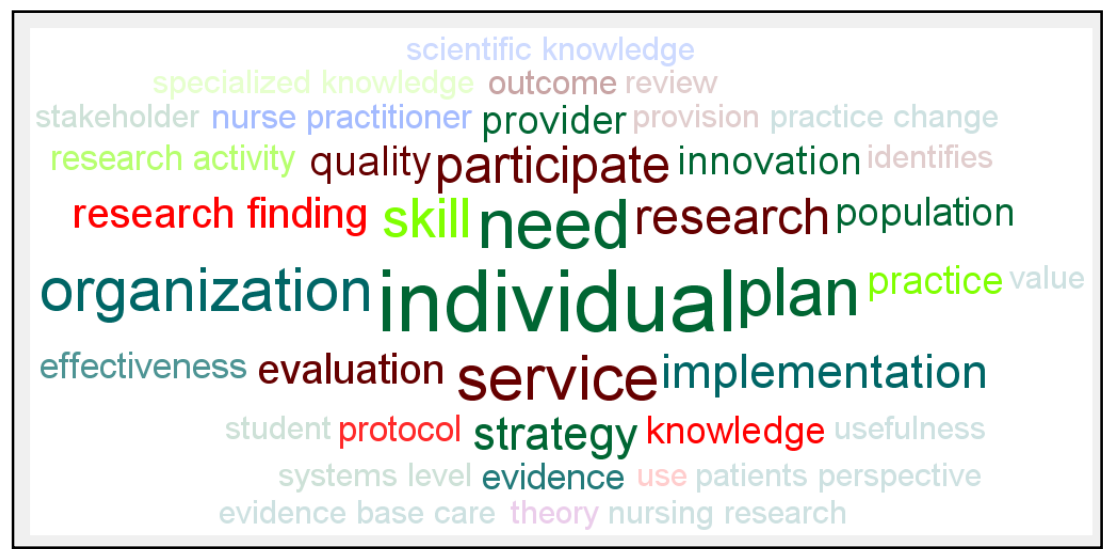

Image x.7. Core elements of Betweeness centrality for Domain 4

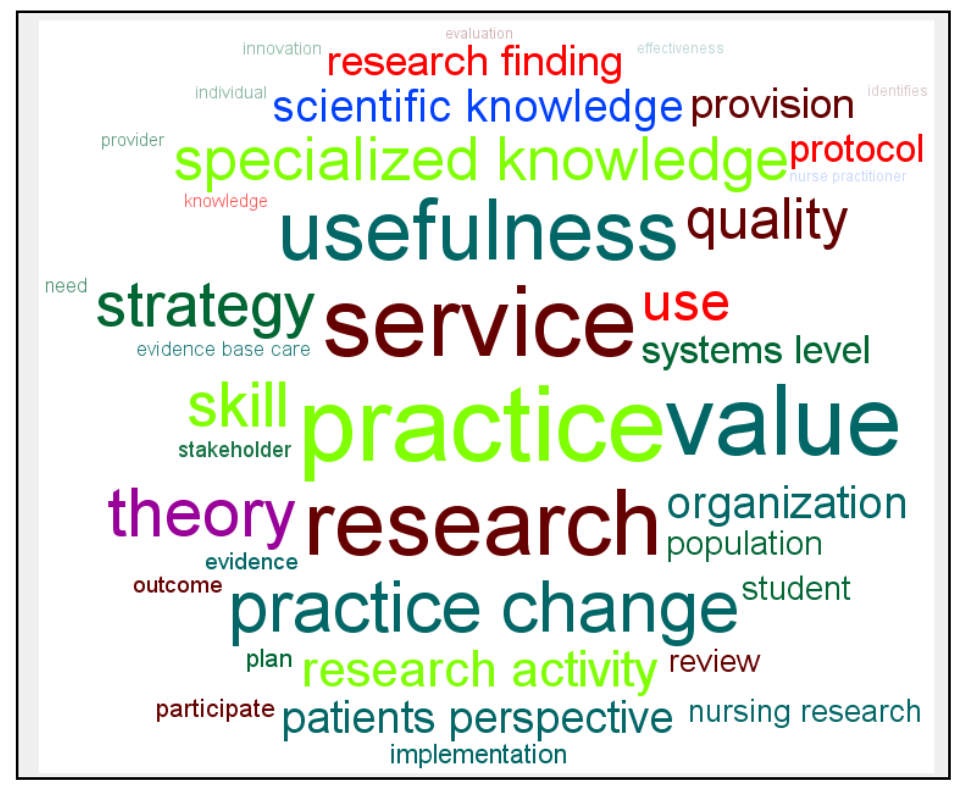

Image x.8. Core elements of Eigenvector centrality for Domain 4 
Finally, about Domain 5: Publication and Professional Leadership that contains 114 words, the analysis shows 10 clusters with the next critical nodes: Leadership; Practice; Care; Evaluation; Professional Context and Critique Health Care Policy; Partnership and System; Responsibility; Educator.

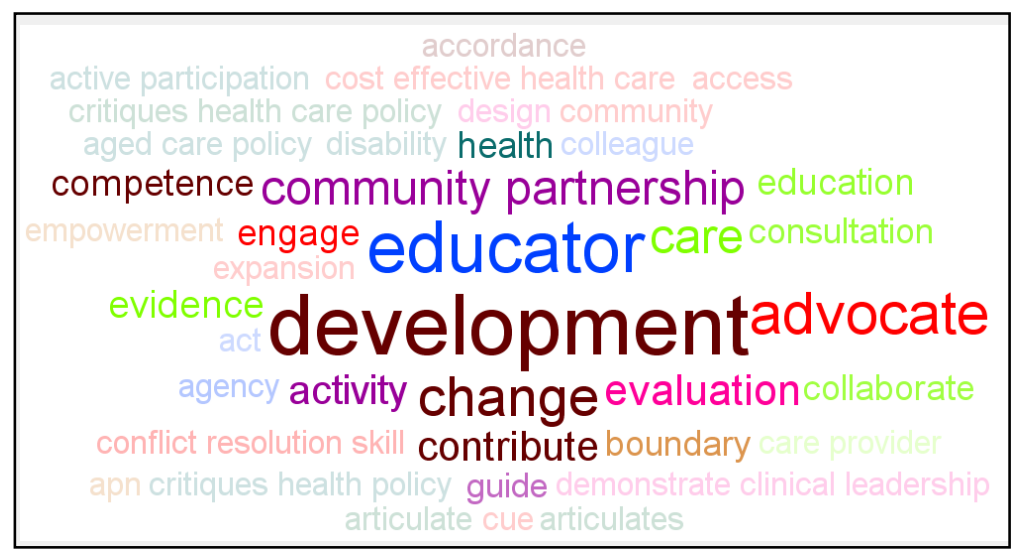

Image x.9. Core elements of Betweeness centrality for Domain 8

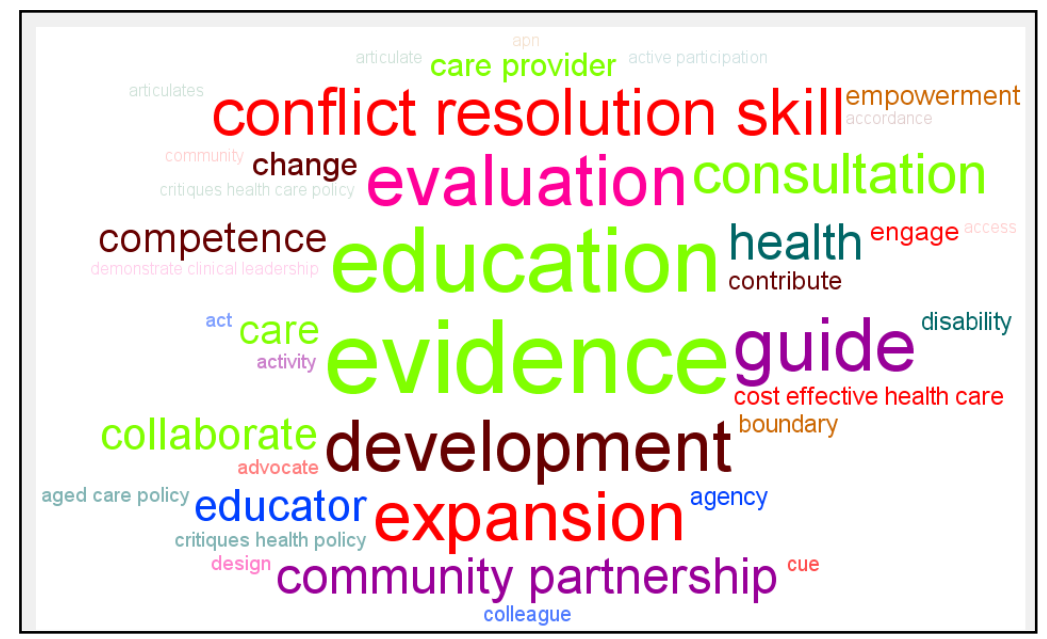

Image x.10. Core elements of Eigenvector centrality for Domain 5 